\title{
A HILBERT INEQUALITY AND AN EULER-MACLAURIN SUMMATION FORMULA
}

\author{
MARIO KRNIĆ ${ }^{\Xi 1}$ and JOSIP PEČARIĆ ${ }^{2}$
}

(Received 12 April, 2006)

\begin{abstract}
We obtain a generalized discrete Hilbert and Hardy-Hilbert inequality with non-conjugate parameters by means of an Euler-Maclaurin summation formula. We derive some general results for homogeneous functions and compare our findings with existing results. We improve some earlier results and apply the results to some special homogeneous functions.

2000 Mathematics subject classification: primary 26D 15.

Keywords and phrases: inequalities, Hilbert's inequality, Bernoulli numbers, Bernoulli polynomials, Euler-Maclaurin summation formula, homogeneous function, kernel, weight function, equivalent inequalities.
\end{abstract}

\section{Introduction}

Let $\left\{a_{n}\right\}$ and $\left\{b_{n}\right\}$ be two non-negative sequences and $1 / p+1 / q=1, p>1$. If $0<\sum_{n=0}^{\infty} a_{n}{ }^{p}<\infty$ and $0<\sum_{n=0}^{\infty} b_{n}{ }^{q}<\infty$, then

$$
\sum_{m=0}^{\infty} \sum_{n=0}^{\infty} \frac{a_{m} b_{n}}{m+n+1}<\frac{\pi}{\sin (\pi / p)}\left(\sum_{n=0}^{\infty} a_{n}{ }^{p}\right)^{1 / p}\left(\sum_{n=0}^{\infty} b_{n}{ }^{q}\right)^{1 / q},
$$

where the constant factor $\pi \sin ^{-1}(\pi / p)$ is the best possible. This is a sharp version of Hilbert's famous theorem for double series, which is important in analysis and its applications. Although classical, inequality (1.1) has attracted the interest of numerous mathematicians and has been generalized in many different ways. For more details see [3].

\footnotetext{
${ }^{1}$ Department of Mathematics, University of Zagreb, Bijenička cesta 30, 10000 Zagreb, Croatia; e-mail: kmic@math.hr.

${ }^{2}$ Faculty of Textile Technology, University of Zagreb, Pierottijeva 6, 10000 Zagreb, Croatia; e-mail: pecaric@hazu.hr.
}

(C) Australian Mathematical Society 2007, Serial-fee code 1446-1811/07 
It is very interesting that one can extend Hilbert's inequality by using numerical analysis. More precisely, one can obtain some extensions by using an Euler-Maclaurin summation formula.

Jichang and Debnath recently obtained the following result using an Euler-Maclaurin summation formula:

THEOREM A. Let $\left\{a_{n}\right\}$ and $\left\{b_{n}\right\}$ be two non-negative sequences and $1 / p+1 / q=1$, with $p>1$. Further, let $0<\sum_{n=0}^{\infty}(n+\mu)^{1-s} a_{n}{ }^{p}<\infty$ and $0<\sum_{n=0}^{\infty}(n+$ $\mu)^{1-s} b_{n}{ }^{q}<\infty$, where $1 / 2 \leq \mu<(1 / 2) \min \{p, q\}$, and let $K(x, y)$ be a non-negative and symmetrical homogeneous function of degree $-s(s>0)$. If $K(1, y)$ has its first four derivatives continuous on $(0, \infty)$, and $(-1)^{n}\left(\partial^{n} K / \partial y^{n}\right)(1, y) \geq 0$ and $\left(\partial^{m} K / \partial y^{m}\right)(1, y) y^{-2 \mu / r} \rightarrow 0, y \rightarrow \infty$ for $m=0,1$, with

$$
I(r, \mu)=\int_{0}^{\infty} K(1, u) u^{-2 \mu / r} d u<\infty, \quad r=p, q,
$$

then

$$
\begin{aligned}
\sum_{m=0}^{\infty} \sum_{n=0}^{\infty} & K(m+\mu, n+\mu) a_{m} b_{n} \\
< & \left\{\sum_{m=0}^{\infty}\left[I(q, \mu)-\phi_{q}(m, s, \mu)\right](m+\mu)^{1-s} a_{m}{ }^{p}\right\}^{1 / p} \\
& \times\left\{\sum_{m=0}^{\infty}\left[I(p, \mu)-\phi_{p}(m, s, \mu)\right](m+\mu)^{1-s} b_{m}{ }^{q}\right\}^{1 / q},
\end{aligned}
$$

where

$$
\begin{aligned}
\phi_{r}(m, s, \mu)= & \left(\frac{\mu}{m+\mu}\right)^{1-2 \mu / r}\left\{K\left(1, \frac{\mu}{m+\mu}\right)\left[\frac{1}{1-2 \mu / r}-\frac{1}{2 \mu}\left(1+\frac{1}{3 r}\right)\right]\right. \\
& \left.-\frac{1}{24 \mu(m+\mu)} \frac{\partial K}{d y}\left(1, \frac{\mu}{m+\mu}\right)\right\}>0,
\end{aligned}
$$

and $r=p, q$.

Obviously, by putting the kernel $K(m, n)=(m+n)^{-1}$ and $\mu=1 / 2$, Inequality (1.2) becomes the improvement of Inequality (1.1).

The main purpose of this paper is a generalization of Inequality (1.2) from Theorem $\mathrm{A}$, as well as the equivalent form of that inequality which we usually call the Hardy-Hilbert inequality (see [3]). One possibility of generalizing it is by extending it to the case of non-conjugate parameters. We shall also compare our results with some previously known from the literature and obtain some improvements of our results from [6] and [5]. 
Throughout this paper $p$ and $q$ indicate real parameters such that $p>1, q>1$ and $1 / p+1 / q \geq 1$. Further, let $p^{\prime}$ and $q^{\prime}$ satisfy $1 / p+1 / p^{\prime}=1$ and $1 / q+1 / q^{\prime}=1$. It is obvious that $p^{\prime}, q^{\prime}>1$. We define $\lambda:=1 / p^{\prime}+1 / q^{\prime}$ and it follows that $0<\lambda \leq 1$ ( $\lambda=1$ gives the case of conjugate exponents). The following result can be found in $[3]$ :

THEOREM B. Let $\left\{a_{n}\right\}$ and $\left\{b_{n}\right\}$ be two non-negative sequences. Under the above definitions, the following inequality holds:

$$
\sum_{m=1}^{\infty} \sum_{n=1}^{\infty} \frac{a_{m} b_{n}}{(m+n)^{\lambda}} \leq K\left(\sum_{m=1}^{\infty} a_{m}{ }^{p}\right)^{1 / p}\left(\sum_{n=1}^{\infty} b_{n}{ }^{q}\right)^{1 / q},
$$

where the constant $K$ depends on $p$ and $q$ only.

Hardy, Littlewood and Pólya did not give a specific value for the constant $K$ in the previous theorem. An alternative proof by Levin, ([8]) established that $K=$ $B^{\lambda}\left(1 / \lambda p^{\prime}, 1 / \lambda q^{\prime}\right)$, where $B$ is a beta function, suffices but the paper did not decide whether this was the best possible constant. This question remains open. The inequality (1.3) was also generalized by Bonsall (see [2]).

The main idea, which was used by the above mentioned authors, is to reduce the case of two non-conjugate parameters to the case of three conjugate parameters. We shall also use that idea in our results. The techniques that will be used in the proofs are mainly based on classical real analysis, especially on Hölder's inequality and on an Euler-Maclaurin summation formula.

\section{Some lemmas}

In order to verify our main results, we need the following lemmas. By using an Euler-Maclaurin summation formula (see [7]) we have the following results.

LEMMA 2.1. Let $f$ have its first four derivatives continuous on $[M, N]$ and $f^{(4)}(x) \geq 0, x \in[M, N]$. Then the following inequality holds:

$$
\sum_{k=M}^{N} f(k)<\int_{M}^{N} f(x) d x+\frac{1}{2}[f(M)+f(N)]+\frac{1}{12}\left[f^{\prime}(N)-f^{\prime}(M)\right] .
$$

ProOF. By the Euler-Maclaurin summation formula in [7], we have

$$
\begin{aligned}
\sum_{k=M}^{N} f(k)= & \int_{M}^{N} f(x) d x+\frac{1}{2}[f(M)+f(N)]+\frac{1}{12}\left[f^{\prime}(N)-f^{\prime}(M)\right] \\
& +\frac{1}{24} \int_{M}^{N} f^{(4)}(t)\left(B_{4}-B_{4}(t-\lfloor t\rfloor)\right) d t
\end{aligned}
$$


where $B_{k}, B_{k}(t)$ are the Bernoulli numbers and the Bernoulli polynomials, respectively. Since $B_{4}=-1 / 30$ and the sign of $B_{4}-B_{4}(t-\lfloor t\rfloor)$ is the same as $B_{4}$, we obtain the inequality (2.1).

RemarK 1. If we put $M=0$ and let $N \rightarrow \infty$, under the assumptions $f(x)$, $f^{\prime}(x) \rightarrow 0$, when $x \rightarrow \infty$, one obtains the inequality

$$
\sum_{k=0}^{\infty} f(k)<\int_{0}^{\infty} f(t) d t+\frac{1}{2} f(0)-\frac{1}{12} f^{\prime}(0)
$$

as in [4].

We now establish the following two results concerning homogeneous functions of negative degree. More precisely, $K:(0, \infty) \times(0, \infty) \mapsto \mathbb{R}$ is a non-negative differentiable homogeneous function of degree $-s, s>0$. We define

$$
k(\alpha):=\int_{0}^{\infty} K(1, u) u^{-\alpha} d u,
$$

and suppose that $k(\alpha)<\infty$ for $\min \{1-s, 0\}<\alpha<\max \{1,2-s\}$. Under these assumptions we have the following result.

LEMMA 2.2. Let $K:(0, \infty) \times(0, \infty) \mapsto \mathbb{R}$ be a non-negative, four times continuously differentiable homogeneous function of degree $-s, s>0$, such that

$$
\begin{gathered}
(-1)^{n} \frac{\partial^{n} K}{\partial y^{n}}(x, y)>0, \quad n=1,2,3,4 \quad \text { and } \\
\lim _{y \rightarrow \infty} K(x, y)=\lim _{y \rightarrow \infty} \frac{\partial K}{\partial y}(x, y)=0 .
\end{gathered}
$$

We define

$$
\Theta_{q^{\prime}}(m, s, \mu):=(m+\mu)^{q^{\prime} A_{1}} \sum_{n=0}^{\infty} K(m+\mu, n+\mu)(n+\mu)^{-q^{\prime} A_{2}},
$$

where $0 \leq A_{2}<1 / q^{\prime}$ and $\mu \geq 1 / 2$. Then the following inequality holds:

$$
\Theta_{q^{\prime}}(m, s, \mu)<(m+\mu)^{1-s+q^{\prime}\left(A_{1}-A_{2}\right)}\left[k\left(q^{\prime} A_{2}\right)-\theta_{q^{\prime}}(m, s, \mu)\right],
$$

where

$$
\begin{aligned}
\theta_{q^{\prime}}(m, s, \mu)= & \left(\frac{\mu}{m+\mu}\right)^{1-q^{\prime} A_{2}}\left\{K\left(1, \frac{\mu}{m+\mu}\right)\left[\frac{1}{1-q^{\prime} A_{2}}-\frac{1}{2 \mu}\left(1+\frac{q^{\prime} A_{2}}{6 \mu}\right)\right]\right. \\
& \left.-\frac{1}{24 \mu(m+\mu)} \frac{\partial K}{d y}\left(1, \frac{\mu}{m+\mu}\right)\right\} .
\end{aligned}
$$


Proof. Let $f$ be the function defined by

$$
f(y):=K(m+\mu, y+\mu) \frac{(m+\mu)^{q^{\prime} A_{1}}}{(y+\mu)^{q^{\prime} A_{2}}} .
$$

Since $\Theta_{q^{\prime}}(m, s, \mu)=\sum_{n=0}^{\infty} f(n)$, we obtain, using Lemma 2.1, the inequality

$$
\Theta_{q^{\prime}}(m, s, \mu)<(m+\mu)^{1-s+q^{\prime}\left(A_{1}-A_{2}\right)}\left[k\left(q^{\prime} A_{2}\right)-\omega_{q^{\prime}}(m, s, \mu)\right],
$$

where

$$
\begin{aligned}
\omega_{q^{\prime}}(m, s, \mu)= & \int_{0}^{\mu /(m+\mu)} K(1, t) t^{-q^{\prime} A_{2}} d t \\
& -\left(\frac{1}{2 \mu}+\frac{q^{\prime} A_{2}}{12 \mu^{2}}\right)\left(\frac{\mu}{m+\mu}\right)^{1-q^{\prime} A_{2}} K\left(1, \frac{\mu}{m+\mu}\right) \\
& +\frac{1}{12 \mu^{2}}\left(\frac{\mu}{m+\mu}\right)^{2-q^{\prime} A_{2}} \frac{\partial K}{d y}\left(1, \frac{\mu}{m+\mu}\right) .
\end{aligned}
$$

Furthermore, if we apply the partial integration formula twice, we have

$$
\begin{aligned}
\int_{0}^{\mu /(m+\mu)} K(1, t) t^{-q^{\prime} A_{2}} d t= & \frac{1}{1-q^{\prime} A_{2}}\left(\frac{\mu}{m+\mu}\right)^{1-q^{\prime} A_{2}} K\left(1, \frac{\mu}{m+\mu}\right) \\
& -\frac{(\mu /(m+\mu))^{2-q^{\prime} A_{2}}}{\left(1-q^{\prime} A_{2}\right)\left(2-q^{\prime} A_{2}\right)} \frac{\partial K}{\partial y}\left(1, \frac{\mu}{m+\mu}\right) \\
& +\frac{1}{\left(1-q^{\prime} A_{2}\right)\left(2-q^{\prime} A_{2}\right)} \int_{0}^{\mu /(m+\mu)} \frac{\partial^{2} K}{\partial y^{2}}(1, t) t^{2-q^{\prime} A_{2}} d t
\end{aligned}
$$

Since the last term in the previous equality is non-negative and since it is easy to verify that

$$
\frac{1}{\left(1-q^{\prime} A_{2}\right)\left(2-q^{\prime} A_{2}\right)}-\frac{1}{12 \mu^{2}}>\frac{1}{24 \mu^{2}},
$$

for $0<q^{\prime} A_{2}<1$ and $\mu \geq 1 / 2$, we obtain the inequality $\omega_{q^{\prime}}(m, s, \mu)>\theta_{q^{\prime}}(m, s, \mu)$, which completes the proof.

REMARK 2. Under the assumptions of Lemma 2.2, the function $\theta_{q^{\prime}}(m, s, \mu)$ is non-negative.

Similarly, if we differentiate with respect to $x$ we obtain the following result.

LEMMA 2.3. Let $K:(0, \infty) \times(0, \infty) \mapsto \mathbb{R}$ be a non-negative, four times continuously differentiable homogeneous function of degree $-s, s>0$, such that

$$
\begin{gathered}
(-1)^{n} \frac{\partial^{n} K}{\partial x^{n}}(x, y)>0, \quad n=1,2,3,4 \quad \text { and } \\
\lim _{x \rightarrow \infty} K(x, y)=\lim _{x \rightarrow \infty} \frac{\partial K}{\partial x}(x, y)=0 .
\end{gathered}
$$


We define

$$
\Theta_{p^{\prime}}(n, s, \mu):=(n+\mu)^{p^{\prime} A_{2}} \sum_{m=0}^{\infty} K(m+\mu, n+\mu)(m+\mu)^{-p^{\prime} A_{1}},
$$

where $0 \leq A_{1}<1 / p^{\prime}$ and $\mu \geq 1 / 2$. Then the following inequality holds:

$$
\Theta_{p^{\prime}}(n, s, \mu)<(n+\mu)^{1-s+p^{\prime}\left(A_{2}-A_{1}\right)}\left[k\left(2-s-p^{\prime} A_{1}\right)-\theta_{p^{\prime}}(n, s, \mu)\right],
$$

where

$$
\begin{aligned}
\theta_{p^{\prime}}(n, s, \mu)= & \left(\frac{\mu}{n+\mu}\right)^{1-p^{\prime} A_{1}}\left\{K\left(\frac{\mu}{n+\mu}, 1\right)\left[\frac{1}{1-p^{\prime} A_{1}}-\frac{1}{2 \mu}\left(1+\frac{p^{\prime} A_{1}}{6 \mu}\right)\right]\right. \\
& \left.-\frac{1}{24 \mu(n+\mu)} \frac{\partial K}{d x}\left(\frac{\mu}{n+\mu}, 1\right)\right\} .
\end{aligned}
$$

REMARK 3. Similarly as before, under the assumptions of Lemma 2.3, the function $\theta_{p^{\prime}}(n, s, \mu)$ is non-negative.

\section{Main results}

In this section, we shall prove our main theorem for homogeneous functions, which is an extension of Theorem A from the introduction, and we also establish some important corollaries. First of all, let's say that we suppose that all the series converge and that they are not identically equal to zero. Under these assumptions we have the following result.

THEOREM 3.1. Let $K:(0, \infty) \times(0, \infty) \mapsto \mathbb{R}$ be a non-negative, four times continuously differentiable homogeneous function of degree $-s$ which satisfies constraints (2.2) and (2.4). Then the following inequalities hold and are equivalent:

$$
\begin{aligned}
\sum_{m=0}^{\infty} & \sum_{n=0}^{\infty} K^{\lambda}(m+\mu, n+\mu) a_{m} b_{n} \\
< & \left\{\sum_{m=0}^{\infty}(m+\mu)^{p(1-s) / q^{\prime}+p\left(A_{1}-A_{2}\right)}\left[k\left(q^{\prime} A_{2}\right)-\theta_{q^{\prime}}(m, s, \mu)\right]^{p / q^{\prime}} a_{m}{ }^{p}\right\}^{1 / p} \\
& \times\left\{\sum_{n=0}^{\infty}(n+\mu)^{q(1-s) / p^{\prime}+q\left(A_{2}-A_{1}\right)}\left[k\left(2-s-p^{\prime} A_{1}\right)-\theta_{p^{\prime}}(n, s, \mu)\right]^{q / p^{\prime}} b_{n}^{q}\right\}^{1 / q}
\end{aligned}
$$


and

$$
\begin{aligned}
& \left\{\sum_{n=0}^{\infty}(n+\mu)^{q^{\prime}\left(A_{1}-A_{2}\right)+q^{\prime}(s-1) / p^{\prime}}\left[k\left(2-s-p^{\prime} A_{1}\right)-\theta_{p^{\prime}}(n, s, \mu)\right]^{-q^{\prime} / p^{\prime}}\right. \\
& \left.\quad \times\left[\sum_{m=0}^{\infty} K^{\lambda}(m+\mu, n+\mu) a_{m}\right]^{q^{\prime}}\right\}^{1 / q^{\prime}} \\
& \quad<\left\{\sum_{m=0}^{\infty}(m+\mu)^{p(1-s) / q^{\prime}+p\left(A_{1}-A_{2}\right)}\left[k\left(q^{\prime} A_{2}\right)-\theta_{q^{\prime}}(m, s, \mu)\right]^{p / q^{\prime}} a_{m}{ }^{p}\right\}^{1 / p},
\end{aligned}
$$

for any $A_{1} \in\left(0,1 / p^{\prime}\right), A_{2} \in\left(0,1 / q^{\prime}\right)$ and $\mu \geq 1 / 2$, where the functions $\theta_{q^{\prime}}(m, s, \mu)$ and $\theta_{p^{\prime}}(n, s, \mu)$ are defined in Lemmas 2.2 and 2.3 , respectively.

PROOF. The left-hand side of Inequality (3.1) can be expressed in the following form:

$$
\begin{aligned}
\sum_{m=0}^{\infty} \sum_{n=0}^{\infty} & K^{\lambda}(m+\mu, n+\mu) a_{m} b_{n} \\
= & \sum_{m=0}^{\infty} \sum_{n=0}^{\infty}\left[K(m+\mu, n+\mu) \frac{(m+\mu)^{p A_{1}}}{(n+\mu)^{q^{\prime} A_{2}}} F^{p-q^{\prime}}(m) a_{m}{ }^{p}\right]^{1 / q^{\prime}} \\
& \times\left[K(m+\mu, n+\mu) \frac{(n+\mu)^{q A_{2}}}{(m+\mu)^{p^{\prime} A_{1}}} G^{q-p^{\prime}}(n) b_{n}^{q}\right]^{1 / p^{\prime}} \\
& \times\left[(m+\mu)^{p A_{1}} F^{p}(m)(n+\mu)^{q A_{2}} G^{q}(n) a_{m}{ }^{p} b_{n}^{q}\right]^{1-\lambda}
\end{aligned}
$$

where the functions $F$ and $G$ are defined by

$$
\begin{aligned}
& F(m)=\left[\sum_{n=0}^{\infty} K(m+\mu, n+\mu)(n+\mu)^{-q^{\prime} A_{2}}\right]^{1 / q^{\prime}} \text { and } \\
& G(n)=\left[\sum_{m=0}^{\infty} K(m+\mu, n+\mu)(m+\mu)^{-p^{\prime} A_{1}}\right]^{1 / p^{\prime}}
\end{aligned}
$$

Now, by applying Hölder's inequality with the conjugate parameters $p^{\prime}, q^{\prime}$ and $1 /(1-\lambda)$ to (3.3) we obtain the inequality

$$
\begin{aligned}
& \sum_{m=0}^{\infty} \sum_{n=0}^{\infty} K^{\lambda}(m+\mu, n+\mu) a_{m} b_{n} \\
& \quad<\left[\sum_{m=0}^{\infty} \Theta_{q^{\prime}}(m, s, \mu)^{p / q^{\prime}} a_{m}{ }^{p}\right]^{1 / p}\left[\sum_{n=0}^{\infty} \Theta_{p^{\prime}}(n, s, \mu)^{q / p^{\prime}} b_{n}{ }^{q}\right]^{1 / q},
\end{aligned}
$$


where the weight functions $\Theta_{q^{\prime}}(m, s, \mu)$ and $\Theta_{p^{\prime}}(n, s, \mu)$ are given by (2.3) and (2.5), respectively. Now, the result follows from Lemmas 2.2 and 2.3.

It remains to prove Inequality (3.2) and the equivalence of Inequalities (3.1) and (3.2). If we put the sequence

$$
\begin{aligned}
b_{n}= & (n+\mu)^{q^{\prime}\left(A_{1}-A_{2}\right)+q^{\prime}(s-1) / p^{\prime}}\left[k\left(2-s-p^{\prime} A_{1}\right)-\theta_{p^{\prime}}(n, s, \mu)\right]^{-q^{\prime} / p^{\prime}} \\
& \times\left[\sum_{m=0}^{\infty} K^{\lambda}(m+\mu, n+\mu) a_{m}\right]^{q^{\prime} / q}
\end{aligned}
$$

in the inequality (3.1), we easily obtain the inequality (3.2).

Otherwise, let's suppose that the inequality (3.2) is valid. By using Hölder's inequality with conjugate parameters $q$ and $q^{\prime}$, we have

$$
\begin{aligned}
\sum_{m=0}^{\infty} \sum_{n=0}^{\infty} & K^{\lambda}(m+\mu, n+\mu) a_{m} b_{n} \\
= & \sum_{n=0}^{\infty}(n+\mu)^{(1-s) / p^{\prime}+A_{2}-A_{1}}\left[k\left(2-s-p^{\prime} A_{1}\right)-\theta_{p^{\prime}}(n, s, \mu)\right]^{1 / p^{\prime}} b_{n} \\
\times & {\left[(n+\mu)^{A_{1}-A_{2}+(s-1) / p^{\prime}}\left[k\left(2-s-p^{\prime} A_{1}\right)-\theta_{p^{\prime}}(n, s, \mu)\right]^{-1 / p^{\prime}}\right.} \\
& \left.\times\left(\sum_{m=0}^{\infty} K^{\lambda}(m+\mu, n+\mu) a_{m}\right)\right] \\
\leq & {\left.\left[\sum_{n=0}^{\infty}(n+\mu)^{q(1-s) / p^{\prime}+q\left(A_{2}-A_{1}\right)}\left[k\left(2-s-p^{\prime} A_{1}\right)-\theta_{p^{\prime}}(n, s, \mu)\right]^{q / p^{\prime}} b_{n}\right]^{q}\right]^{1 / q} } \\
& \times\left[\sum_{n=0}^{\infty}(n+\mu)^{q^{\prime}\left(A_{1}-A_{2}\right)+q^{\prime}(s-1) / p^{\prime}}\left[k\left(2-s-p^{\prime} A_{1}\right)-\theta_{p^{\prime}}(n, s, \mu)\right]^{-q^{\prime} / p^{\prime}}\right. \\
\times & \left.\left(\sum_{m=0}^{\infty} K^{\lambda}(m+\mu, n+\mu) a_{m}\right)^{q^{\prime}}\right]^{1 / q^{\prime}},
\end{aligned}
$$

and the result follows from the inequality (3.2). So, the inequalities (3.1) and (3.2) are equivalent which completes the proof.

Now, if we put $A_{1}=A_{2}=2 \mu / p^{\prime} q^{\prime}$ in the previous theorem, we obtain the extension of Theorem $A$ to the case of non-conjugate parameters.

COROllaRY 3.2. Let $K:(0, \infty) \times(0, \infty) \mapsto \mathbb{R}$ be non-negative, four times continuously differentiable homogeneous function of degree $-s$ which satisfy conditions 
(2.2) and (2.4). Then the following inequalities hold and are equivalent:

$$
\begin{aligned}
\sum_{m=0}^{\infty} \sum_{n=0}^{\infty} K^{\lambda}(m+\mu, n+\mu) a_{m} b_{n} \\
< \\
\quad\left\{\sum_{m=0}^{\infty}(m+\mu)^{p / q^{\prime}(1-s)}\left[k\left(\frac{2 \mu}{p^{\prime}}\right)-\phi_{p^{\prime}}(m, s, \mu)\right] a_{m}{ }^{p}\right\}^{1 / p} \\
\times\left\{\sum_{n=0}^{\infty}(n+\mu)^{q / p^{\prime}(1-s)}\left[k\left(2-s-\frac{2 \mu}{q^{\prime}}\right)-\phi_{q^{\prime}}(m, s, \mu)\right] b_{n}{ }^{q}\right\}^{1 / q}
\end{aligned}
$$

and

$$
\begin{aligned}
& \left\{\sum_{n=0}^{\infty}(n+\mu)^{q^{\prime}(s-1) / p^{\prime}}\left[k\left(2-s-\frac{2 \mu}{q^{\prime}}\right)-\phi_{q^{\prime}}(n, s, \mu)\right]^{-q^{\prime} / p^{\prime}}\right. \\
& \left.\quad \times\left[\sum_{m=0}^{\infty} K^{\lambda}(m+\mu, n+\mu) a_{m}\right]^{q^{\prime}}\right\}^{1 / q^{\prime}} \\
& \quad<\left\{\sum_{m=0}^{\infty}(m+\mu)^{p(1-s) / q^{\prime}}\left[k\left(\frac{2 \mu}{p^{\prime}}\right)-\theta_{p^{\prime}}(m, s, \mu)\right]^{p / q^{\prime}} a_{m}^{p}\right\}^{1 / p},
\end{aligned}
$$

where $1 / 2 \leq \mu<(1 / 2) \min \left\{p^{\prime}, q^{\prime}\right\}$ and the functions $\phi_{p^{\prime}}$ and $\phi_{q^{\prime}}$ are defined as in Theorem A.

REMARK 4. If the function $K:(0, \infty) \times(0, \infty) \mapsto \mathbb{R}$ is homogeneous and symmetrical then $k(2-s-\alpha)=k(\alpha)$. Hence, if $K$ is symmetrical and $\lambda=1$, then $p^{\prime}=q$ and $q^{\prime}=p$, so Inequality (3.4) becomes Inequality (1.2) from Theorem A.

Further, by putting $\lambda=1$ in Theorem 3.1 we obtain the following result.

THEOREM 3.3. Let $K:(0, \infty) \times(0, \infty) \mapsto \mathbb{R}$ be a non-negative, four times continuously differentiable homogeneous function of degree $-s$ which satisfies conditions (2.2) and (2.4). Then the following inequalities hold:

$$
\begin{aligned}
\sum_{m=1}^{\infty} \sum_{n=1}^{\infty} K^{\lambda}(m, n) a_{m} b_{n} \\
< \\
\quad\left\{\sum_{m=1}^{\infty} m^{p(1-s) / q^{\prime}+p\left(A_{1}-A_{2}\right)}\left[k\left(q^{\prime} A_{2}\right)-\varphi_{q^{\prime}}(m, s, \mu)\right]^{p / q^{\prime}} a_{m}{ }^{p}\right\}^{1 / p} \\
\times\left\{\sum_{n=1}^{\infty} n^{q(1-s) / p^{\prime}+q\left(A_{2}-A_{1}\right)}\left[k\left(2-s-p^{\prime} A_{1}\right)-\varphi_{p^{\prime}}(n, s, \mu)\right]^{q / p^{\prime}} b_{n}{ }^{q}\right\}^{1 / q}
\end{aligned}
$$


and

$$
\begin{aligned}
& \left\{\sum_{n=1}^{\infty} n^{q^{\prime}\left(A_{1}-A_{2}\right)+q^{\prime}(s-1) / p^{\prime}}\left[k\left(2-s-p^{\prime} A_{1}\right)-\varphi_{p^{\prime}}(n, s, \mu)\right]^{-q^{\prime} / p^{\prime}}\right. \\
& \left.\quad \times\left[\sum_{m=1}^{\infty} K^{\lambda}(m, n) a_{m}\right]^{q^{\prime}}\right\}^{1 / q^{\prime}} \\
& \quad<\left\{\sum_{m=1}^{\infty} m^{p(1-s) / q^{\prime}+p\left(A_{1}-A_{2}\right)}\left[k\left(q^{\prime} A_{2}\right)-\varphi_{q^{\prime}}(m, s, \mu)\right]^{p / q^{\prime}} a_{m}^{p}\right\}^{1 / p}
\end{aligned}
$$

for any $A_{1} \in\left(0,1 / p^{\prime}\right), A_{2} \in\left(0,1 / q^{\prime}\right)$ and $\mu \geq 1 / 2$, where

$$
\begin{aligned}
& \varphi_{q^{\prime}}(m, s, \mu) \\
& \quad=\left(\frac{1}{m}\right)^{1-q^{\prime} A_{2}}\left\{K\left(1, \frac{1}{m}\right)\left[\frac{1}{1-q^{\prime} A_{2}}-\frac{1}{2}-\frac{q^{\prime} A_{2}}{12}\right]-\frac{1}{24 m} \frac{\partial K}{\partial y}\left(1, \frac{1}{m}\right)\right\}
\end{aligned}
$$

and

$$
\begin{aligned}
& \varphi_{p^{\prime}}(n, s, \mu) \\
& \quad=\left(\frac{1}{n}\right)^{1-p^{\prime} A_{1}}\left\{K\left(\frac{1}{n}, 1\right)\left[\frac{1}{1-p^{\prime} A_{1}}-\frac{1}{2}-\frac{p^{\prime} A_{1}}{12}\right]-\frac{1}{24 n} \frac{\partial K}{\partial x}\left(\frac{1}{n}, 1\right)\right\} .
\end{aligned}
$$

In particular, Inequalities (3.6) and (3.7) are equivalent.

REMARK 5. The functions $\varphi_{p^{\prime}}$ and $\varphi_{q^{\prime}}$ from Theorem 3.3 are non-negative (see Remarks 2 and 3). Inequalities (3.6) and (3.7) are improvements of those in our papers [6] and [5] in the conjugate case.

In the next, we apply our general results to some special choices of homogeneous functions.

\section{Some applications}

We now apply our general results to some special homogeneous functions whose first four partial derivatives satisfy conditions (2.2) and (2.4). First, consider the function $K(x, y)=(x+y)^{-s}$. In this case $k(\alpha)=B(1-\alpha, s+\alpha-1)$, where $B$ is a beta function. We define $b(\alpha):=B(1-\alpha, s+\alpha-1)$ and obtain the following result. 
THEOREM 4.1. The following inequalities hold and are equivalent

$$
\begin{aligned}
\sum_{m=0}^{\infty} \sum_{n=0}^{\infty} & \frac{a_{m} b_{n}}{(m+n+2 \mu)^{\lambda s}} \\
< & \left\{\sum_{m=0}^{\infty}(m+\mu)^{p(1-s) / q^{\prime}+p\left(A_{1}-A_{2}\right)}\left[b\left(q^{\prime} A_{2}\right)-\psi_{q^{\prime}}(m, s, \mu)\right]^{p / q^{\prime}} a_{m}{ }^{p}\right\}^{1 / p} \\
& \times\left\{\sum_{n=0}^{\infty}(n+\mu)^{q(1-s) / p^{\prime}+q\left(A_{2}-A_{1}\right)}\left[b\left(p^{\prime} A_{1}\right)-\psi_{p^{\prime}}(n, s, \mu)\right]^{q / p^{\prime}} b_{n}\right\}^{1 / q}
\end{aligned}
$$

and

$$
\begin{aligned}
& \left\{\sum_{n=0}^{\infty}(n+\mu)^{q^{\prime}\left(A_{1}-A_{2}\right)+q^{\prime}(s-1) / p^{\prime}}\left[b\left(p^{\prime} A_{1}\right)-\psi_{p^{\prime}}(n, s, \mu)\right]^{-q^{\prime} / p^{\prime}}\right. \\
& \left.\quad \times\left[\sum_{m=0}^{\infty} \frac{a_{m}}{(m+n+2 \mu)^{\lambda s}}\right]^{q^{\prime}}\right\}^{1 / q^{\prime}} \\
& \quad<\left\{\sum_{m=0}^{\infty}(m+\mu)^{p(1-s) / q^{\prime}+p\left(A_{1}-A_{2}\right)}\left[b\left(q^{\prime} A_{2}\right)-\psi_{q^{\prime}}(m, s, \mu)\right]^{p / q^{\prime}} a_{m}{ }^{p}\right\}^{1 / p},
\end{aligned}
$$

for any $A_{1} \in\left(\max \left\{(1-s) / p^{\prime}, 0\right\}, 1 / p^{\prime}\right), A_{2} \in\left(\max \left\{(1-s) / q^{\prime}, 0\right\}, 1 / q^{\prime}\right)$ and $\mu \geq 1 / 2$, where

and

$$
\psi_{q^{\prime}}(m, s, \mu)=\left(\frac{\mu}{m+\mu}\right)^{1-q^{\prime} A_{2}} \frac{1}{2^{s}}\left[\frac{1}{1-q^{\prime} A_{2}}-\frac{1}{2 \mu}\left(1+\frac{q^{\prime} A_{2}}{6 \mu}\right)\right]
$$

$$
\psi_{p^{\prime}}(n, s, \mu)=\left(\frac{\mu}{n+\mu}\right)^{1-p^{\prime} A_{1}} \frac{1}{2^{s}}\left[\frac{1}{1-p^{\prime} A_{1}}-\frac{1}{2 \mu}\left(1+\frac{p^{\prime} A_{1}}{6 \mu}\right)\right]
$$

PROOF. The result easily follows by putting $K(x, y)=(x+y)^{-s}$ in Theorem 3.1, and noting that $\psi_{q^{\prime}}(m, s, \mu)<\theta_{q^{\prime}}(m, s, \mu)$ and $\psi_{p^{\prime}}(n, s, \mu)<\theta_{p^{\prime}}(n, s, \mu)$.

REMARK 6. If we put $A_{1}=A_{2}=2 \mu / p^{\prime} q^{\prime}$ in Theorem 4.1 , then, under the assumption $\max \left\{1 / 2,(1-s) / 2 p^{\prime},(1-s) / 2 q^{\prime}\right\}<\mu<\max \left\{p^{\prime} / 2, q^{\prime} / 2\right\}$, we obtain, in the conjugate case, the result from [4].

Further, we consider the function $K(x, y)=\left(x^{s}+y^{s}\right)^{-1}$. For such a function we have $k(\alpha)=(1 / s) B((1-\alpha) / s,(s+\alpha-1) / s)$, where $B$ is a beta function. We define $b_{s}(\alpha):=(1 / s) B((1-\alpha) / s,(s+\alpha-1) / s)$ and obtain the following theorem. 
THEOREM 4.2. The following inequalities hold and are equivalent:

$$
\begin{aligned}
\sum_{m=0}^{\infty} \sum_{n=0}^{\infty} & \frac{a_{m} b_{n}}{\left[(m+\mu)^{s}+(n+\mu)^{s}\right]^{\lambda}} \\
< & \left\{\sum_{m=0}^{\infty}(m+\mu)^{p(1-s) / q^{\prime}+p\left(A_{1}-A_{2}\right)}\left[b_{s}\left(q^{\prime} A_{2}\right)-2^{s-1} \psi_{q^{\prime}}(m, s, \mu)\right]^{p / q^{\prime}} a_{m}{ }^{p}\right\}^{1 / p} \\
& \times\left\{\sum_{n=0}^{\infty}(n+\mu)^{q(1-s) / p^{\prime}+q\left(A_{2}-A_{1}\right)}\left[b_{s}\left(p^{\prime} A_{1}\right)-2^{s-1} \psi_{p^{\prime}}(n, s, \mu)\right]^{q / p^{\prime}} b_{n}{ }^{q}\right\}^{1 / q}
\end{aligned}
$$

and

$$
\begin{aligned}
& \left\{\sum_{n=0}^{\infty}(n+\mu)^{q^{\prime}\left(A_{1}-A_{2}\right)+q^{\prime}(s-1) / p^{\prime}}\left[b_{s}\left(p^{\prime} A_{1}\right)-2^{s-1} \psi_{p^{\prime}}(n, s, \mu)\right]^{-q^{\prime} / p^{\prime}}\right. \\
& \left.\times\left[\sum_{m=0}^{\infty} \frac{a_{m}}{\left[(m+\mu)^{s}+(n+\mu)^{s}\right]^{\lambda}}\right]^{q^{\prime}}\right\}^{1 / q^{\prime}} \\
& \quad<\left\{\sum_{m=0}^{\infty}(m+\mu)^{p(1-s) / q^{\prime}+p\left(A_{1}-A_{2}\right)}\left[b_{s}\left(q^{\prime} A_{2}\right)-2^{s-1} \psi_{q^{\prime}}(m, s, \mu)\right]^{p / q^{\prime}} a_{m}{ }^{p}\right\}^{1 / p},
\end{aligned}
$$

for any $A_{1} \in\left(\max \left\{(1-s) / p^{\prime}, 0\right\}, 1 / p^{\prime}\right), A_{2} \in\left(\max \left\{(1-s) / q^{\prime}, 0\right\}, 1 / q^{\prime}\right)$ and $\mu \geq$ $1 / 2$, where the functions $\psi_{q^{\prime}}(m, s, \mu)$ and $\psi_{p^{\prime}}(n, s, \mu)$ are defined as in the previous theorem.

REMARK 7. Similarly, by putting $A_{1}=A_{2}=2 \mu / p^{\prime} q^{\prime}$ in Theorem 4.2 , under the assumption $\max \left\{1 / 2,(1-s) / 2 p^{\prime},(1-s) / 2 q^{\prime}\right\}<\mu<\max \left\{p^{\prime} / 2, q^{\prime} / 2\right\}$, we obtain the generalization of the result from [4].

It is interesting to put $A_{1}=(1-s / r) / p^{\prime}$ and $A_{2}=(1-s / t) / q^{\prime}$, where $r$ and $t$ are conjugate parameters with $r>1$ and $0<s \leq 1$. Under these assumptions we have

COROLlaRY 4.3. If $1 / r+1 / t=1$, with $r>1$ and $0<s \leq 1$, then the following inequalities hold and are equivalent

$$
\begin{aligned}
\sum_{m=0}^{\infty} \sum_{n=0}^{\infty} & \frac{a_{m} b_{n}}{\left[(m+\mu)^{s}+(n+\mu)^{s}\right]^{\lambda}} \\
< & \left\{\sum_{m=0}^{\infty}(m+\mu)^{p\left(1 / p^{\prime}-s \lambda / r\right)}\left[\frac{\pi}{s \sin (\pi / r)}-2^{s-1} \psi_{q^{\prime}}(m, s, \mu)\right]^{p / q^{\prime}} a_{m}{ }^{p}\right\}^{1 / p} \\
& \times\left\{\sum_{n=0}^{\infty}(n+\mu)^{q\left(1 / q^{\prime}-s \lambda / r\right)}\left[\frac{\pi}{s \sin (\pi / t)}-2^{s-1} \psi_{p^{\prime}}(n, s, \mu)\right]^{q / p^{\prime}} b_{n}^{q}\right\}^{1 / q}
\end{aligned}
$$


and

$$
\begin{aligned}
& \left\{\sum_{n=0}^{\infty}(n+\mu)^{s q^{\prime} \lambda / t-1}\left[\frac{\pi}{s \sin (\pi / t)}-2^{s-1} \psi_{p^{\prime}}(n, s, \mu)\right]^{-q^{\prime} / p^{\prime}}\right. \\
& \left.\quad \times\left[\sum_{m=0}^{\infty} \frac{a_{m}}{\left[(m+\mu)^{s}+(n+\mu)^{s}\right]^{\lambda}}\right]^{q^{\prime}}\right\}^{1 / q^{\prime}} \\
& \quad<\left\{\sum_{m=0}^{\infty}(m+\mu)^{p\left(1 / p^{\prime}-s \lambda / r\right)}\left[\frac{\pi}{s \sin (\pi / r)}-2^{s-1} \psi_{q^{\prime}}(m, s, \mu)\right]^{p / q^{\prime}} a_{m}^{p}\right\}^{1 / p},
\end{aligned}
$$

where the functions $\psi_{q^{\prime}}(m, s, \mu)$ and $\psi_{p^{\prime}}(n, s, \mu)$ are defined in Theorem 4.1.

REMARK 8 . If $\lambda=1$, then we obtain an inequality which is sharper than that in [1].

REMARK 9. Other types of results can be obtained by putting $A_{1}=A_{2}=\frac{2-s}{p^{\prime} q^{\prime}}$, where $p^{\prime}, q^{\prime}$ and $s$ satisfy the constraint

$$
\max \left\{1-\min \left\{\frac{1}{p^{\prime}-1}, \frac{1}{q^{\prime}-1}\right\}, 2-\min \left\{p^{\prime}, q^{\prime}\right\}\right\}<s \leq 2 .
$$

In such a way improve our results from [6] and [5], as well as some previously known results which include the beta function.

\section{References}

[1] Y. Bicheng, "On an extension of Hardy-Hilbert's inequality with some parameters", Math. Inequal. Appl. (to appear).

[2] F. F. Bonsall, "Inequalities with non-conjugate parameters", Quart. J. Math. Oxford (2) 2 (1951) $135-150$.

[3] G. H. Hardy, J. E. Littlewood and G. Pólya, Inequalities (Cambridge Univ. Press, Cambridge, 1952).

[4] K. Jichang and L. Debnath, "On new generalizations of Hilbert's inequality and their applications", Math. Inequal. Appl. 245 (2000) 248-265.

[5] M. Krnić and J. Pečarić, "General Hilbert's and Hardy's inequalities", Math. Inequal. Appl. 8 (2005) 29-51.

[6] M. Krnić and J. Pečarić, "Hilbert's inequalities and their reverses", Publ. Math. Debrecen 67 (2005) 315-331.

[7] V. I. Krylov, Approximate calculation of integrals (Macmillan, New York, 1962).

[8] V. Levin, "On the two parameter extension and analogue of Hilbert's inequality", J. London Math. Soc. 11 (1936) 119-124. 